\title{
Current state of hybrid solutions for aortic arch aneurysms
}

\author{
G. Chad Hughes, Andrew Vekstein \\ Duke University Medical Center, Division of Cardiovascular and Thoracic Surgery, Department of Surgery, Duke University, Durham, NC, USA \\ Correspondence to: G. Chad Hughes, MD. Director, Center for Aortic Disease, Duke University Medical Center, Department of Surgery, Box 3051 \\ DUMC, Durham, NC 27710, USA. Email: gchad.hughes@duke.edu.
}

\begin{abstract}
Since its inception in the early 2000s, hybrid arch repair (HAR) has evolved from a novel approach to a wellestablished treatment modality for aortic arch pathology in appropriately selected patients. HAR procedures have been proposed as a means to circumvent the perioperative morbidity and mortality associated with open total arch replacement. These procedures, all of which remain off-label applications of approved endograft technology, combine more conventional open surgical techniques, to create endograft landing zones, with thoracic endovascular aortic repair to exclude the aortic pathology from the circulation. The current classification system for HAR was proposed in 2013 and consists of three types, designated by the Roman numerals I, II and III. The current system has become outdated, however, with the advent of newer technologies, and herein we propose a new, updated classification system that is more encompassing with regards to the broad array of options available to treat aortic arch disease. Likewise, an institutional algorithm to guide patient and operative selection for HAR is presented. Patients are considered for HAR if they have either high-risk comorbidities or high-risk anatomy, with an important feature of the algorithm being that any decisions about repair strategy should be made by a surgical team with expertise in both open and endovascular techniques. Despite being performed for nearly two decades, the evidence around HAR consists mainly of single center series (level B-C evidence) with no randomized controlled trials. The data suggest HAR to be a safe alternative to open repair with acceptable short and mid-term results. As we as aortic surgeons continue to move towards less invasive approaches, both conventional open and hybrid techniques will remain important tools in the toolbox for arch repair, although the advent of multi-branched arch endografts will almost certainly reduce the extent of open or hybrid repair in many patients and eliminate it altogether in others.
\end{abstract}

Keywords: Hybrid; aortic arch; endovascular; aneurysm; dissection

Submitted Sep 02, 2021. Accepted for publication Oct 27, 2021.

doi: 10.21037/acs-2021-taes-168

View this article at: https://dx.doi.org/10.21037/acs-2021-taes-168

\section{Introduction}

Aortic arch surgery continues to become increasingly common with data from the Society of Thoracic Surgeons Adult Cardiac Surgery Database (STS-ACSD) demonstrating a nearly $25 \%$ increase in case volumes from 2011-2014, a rate of increase faster than that of cardiac surgery as a whole (1). Despite increasing operator experience, however, mortality, stroke, and renal failure rates of $12 \%, 8 \%$ and $9 \%$, respectively, in that study were not significantly improved from an earlier STS-ACSD report spanning the time interval 2004-2009 (2). Further, many patients may be ineligible for conventional arch repair due to advanced age, frailty (3), or multiple comorbidities.

Since the initial description by Czerny and colleagues in 2003 (4) of combined open arch debranching and endovascular exclusion for an arch aneurysm, so-called hybrid arch repair (HAR) procedures have been proposed as a means to circumvent the perioperative morbidity and mortality associated with open total arch replacement (5). These procedures, all of which remain off-label applications of approved endograft technology, combine more conventional open surgical techniques, to create endograft landing 

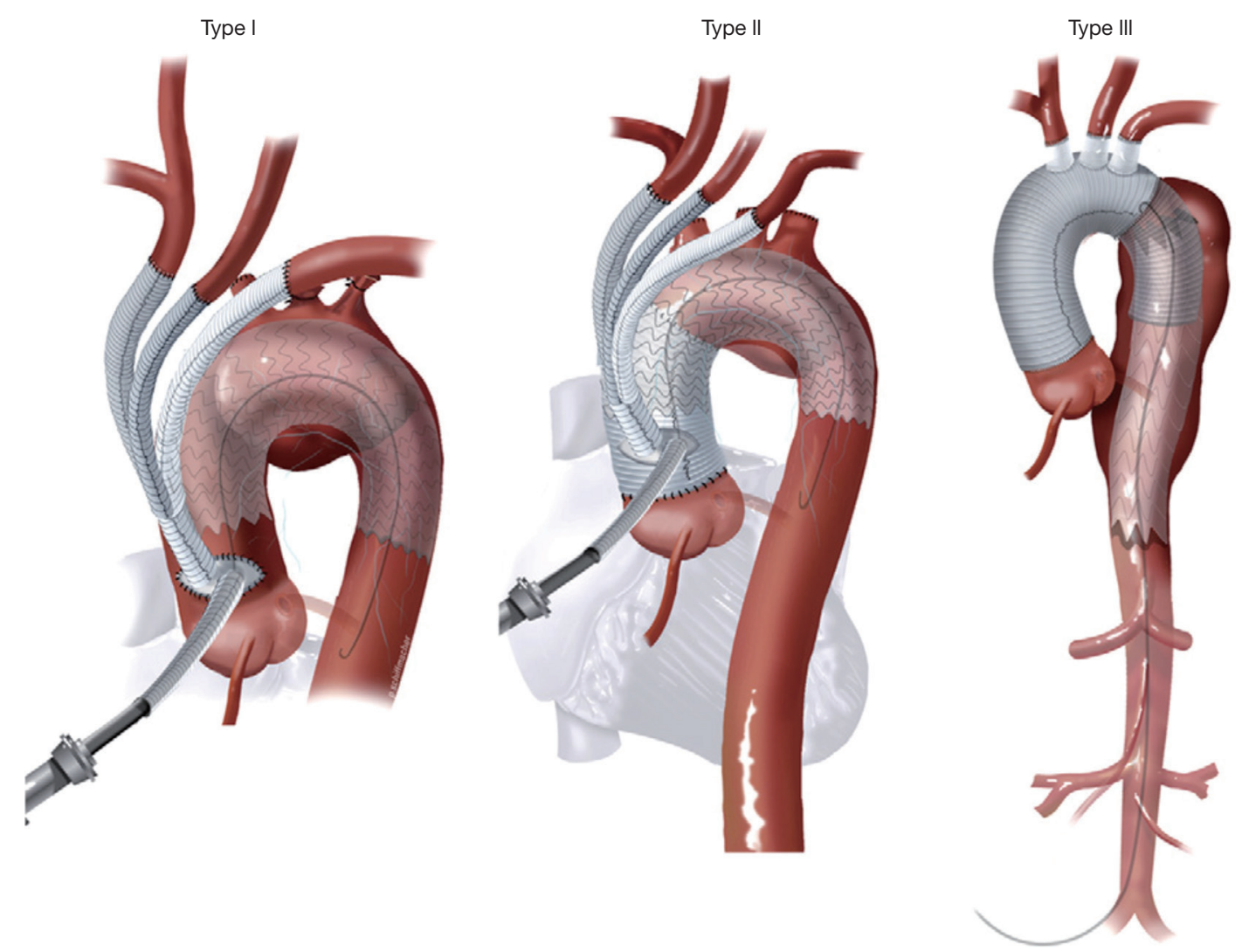

Figure 1 Original HAR classification system proposed in 2013. Reproduced with permission from reference (6). HAR, hybrid arch repair.

zones, with thoracic endovascular aortic repair (TEVAR) to exclude the aortic pathology from the circulation. This paper will review the current state of hybrid solutions for arch repair and propose a new expanded classification system that encompasses all options currently available for HAR.

\section{HAR subtypes \& classification system}

The current classification system for HAR was proposed by Bavaria and colleagues in 2013 (6) and consists of three types, designated by the Roman numerals I, II, and III (Figure 1).

\section{Type I HAR}

Type I HAR (Figure 1) involves debranching of the aortic arch vessels, typically via partial or full sternotomy, using a multi-branched Dacron graft sewn to either the native ascending aorta (native zone 0) or a previously Dacronreplaced ascending aorta (Dacron zone 0), the latter most commonly in the setting of prior type A dissection repair. A type I HAR is generally utilized for patients with midtransverse arch \pm descending pathology, but with $\geq 2 \mathrm{~cm}$ of suitable proximal landing zone (PLZ) in the native ascending aorta (or $\geq 4 \mathrm{~cm}$ if the PLZ consists of Dacronreplaced ascending aorta as detailed below) (7).

In certain cases, the left subclavian artery (LSA) may not be readily accessible via a sternotomy approach due to distal takeoff from the arch, in which case type I HAR may involve only debranching of the innominate and left common carotid arteries from the ascending aorta, with the LSA revascularized via a carotid-subclavian bypass (8) (Figure 2) or transposition (9), carotid-axillary bypass (10), or alternatively via a transthoracic aorta to infraclavicular axillary artery bypass (Figure 3, left panel).

After completion of the arch vessel debranching, the arch pathology is then excluded using commercially available thoracic endografts deployed via a retrograde iliofemoral approach, or less commonly, via an antegrade approach from the ascending aorta. The latter approach typically involves the use of a commercially available arch 


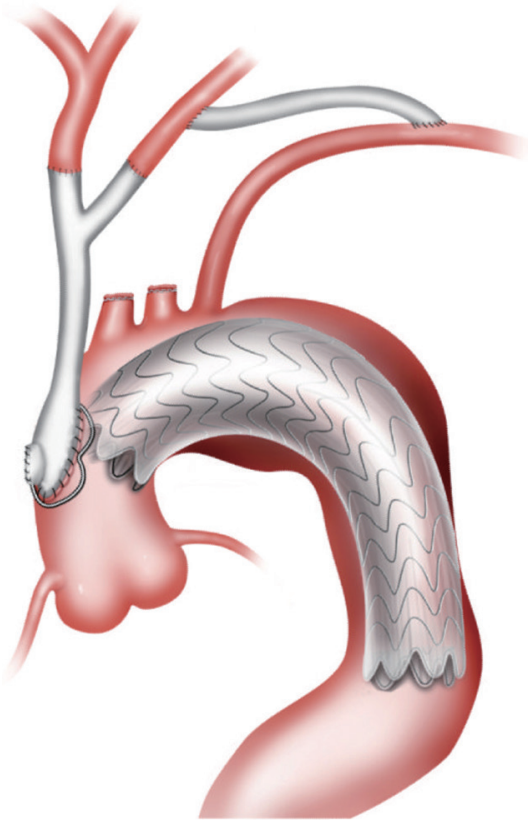

Figure 2 Type I HAR with LSA revascularized via carotidsubclavian bypass. Although not shown in the drawing, the LSA would typically be occluded proximally using endovascular techniques as part of the procedure. HAR, hybrid arch repair; LSA, left subclavian artery.

debranching Dacron graft with an integral limb designed for antegrade stent graft deployment and is performed during the same procedure as the arch debranching (Figure 4). With iliofemoral endograft delivery, however, the TEVAR portion of the procedure is more commonly performed in a staged fashion, typically during the same hospital stay, as detailed below in the section on Operative Considerations. We prefer a staged approach with retrograde iliofemoral endograft delivery and reserve the use of single-stage antegrade deployment cases to those situations in which the patient has inadequate iliofemoral access for retrograde delivery.

\section{Type II HAR}

Type II HAR (Figure 1) involves replacement of the ascending aorta and, most commonly, a portion of the aortic arch with the distal anastomosis frequently in arch zones 1 or 2, along with debranching of the arch vessels similar to type I HAR. This procedure is utilized when no suitable native or Dacron-replaced zone 0 PLZ exists and results in the creation of 4-6 cm PLZ for the second stage TEVAR (11).
Type II HAR is performed in a staged approach, with the first stage proximal aortic replacement and second stage TEVAR procedures usually carried out during a single hospital stay (11).

\section{Type III HAR}

Type III HAR (Figure 1) is similar to type II HAR with the exception that a conventional elephant trunk is created for use as PLZ. This results in the creation of a much longer $(10 \mathrm{~cm})$ PLZ. However, as detailed below, this technique has become unnecessary in our opinion with the commercial availability of Dacron grafts designed for type II HAR, because the distal anastomosis for type II HAR is simpler and requires shorter cardiopulmonary bypass (CPB) and hypothermic circulatory arrest (HCA) times (11). Although not part of the original Bavaria classification system (6), some authors likewise consider the frozen elephant trunk (FET) procedure a variant of type III HAR $(12,13)$. FET involves replacement of the ascending aorta and a portion of the aortic arch, but rather than placing a conventional Dacron elephant trunk graft into the descending thoracic aorta to serve as future PLZ, a thoracic endograft is utilized as the elephant trunk graft and placed into the descending thoracic aorta via the open arch under HCA at the time of arch replacement (Figure 5) (14). The proximal end of the FET endograft is then incorporated into the distal anastomosis of the Dacron graft used to replace the aortic arch.

\section{Zone 1 HAR}

Another type of HAR, not included in the original classification system, is the so-called zone 1 HAR (Figure 6, left panel), which involves cervical arch debranching (right common carotid-left common carotid-left subclavian artery bypass via neck incisions) with PLZ in zone 1 just distal to the innominate artery (16). This is useful for patients with distal arch \pm descending pathology but with $\geq 2 \mathrm{~cm}$ of PLZ distal to the innominate artery (zone 1) (7). The procedure has the advantage of avoiding median sternotomy and the need for $\mathrm{CPB}$ or HCA.

\section{Zone 0 HAR}

Zone $0 \mathrm{HAR}$, also not included in the original classification, involves cervical arch debranching similar to the zone 1 HAR procedure, but with PLZ in zone 0 (Figure 6, right 


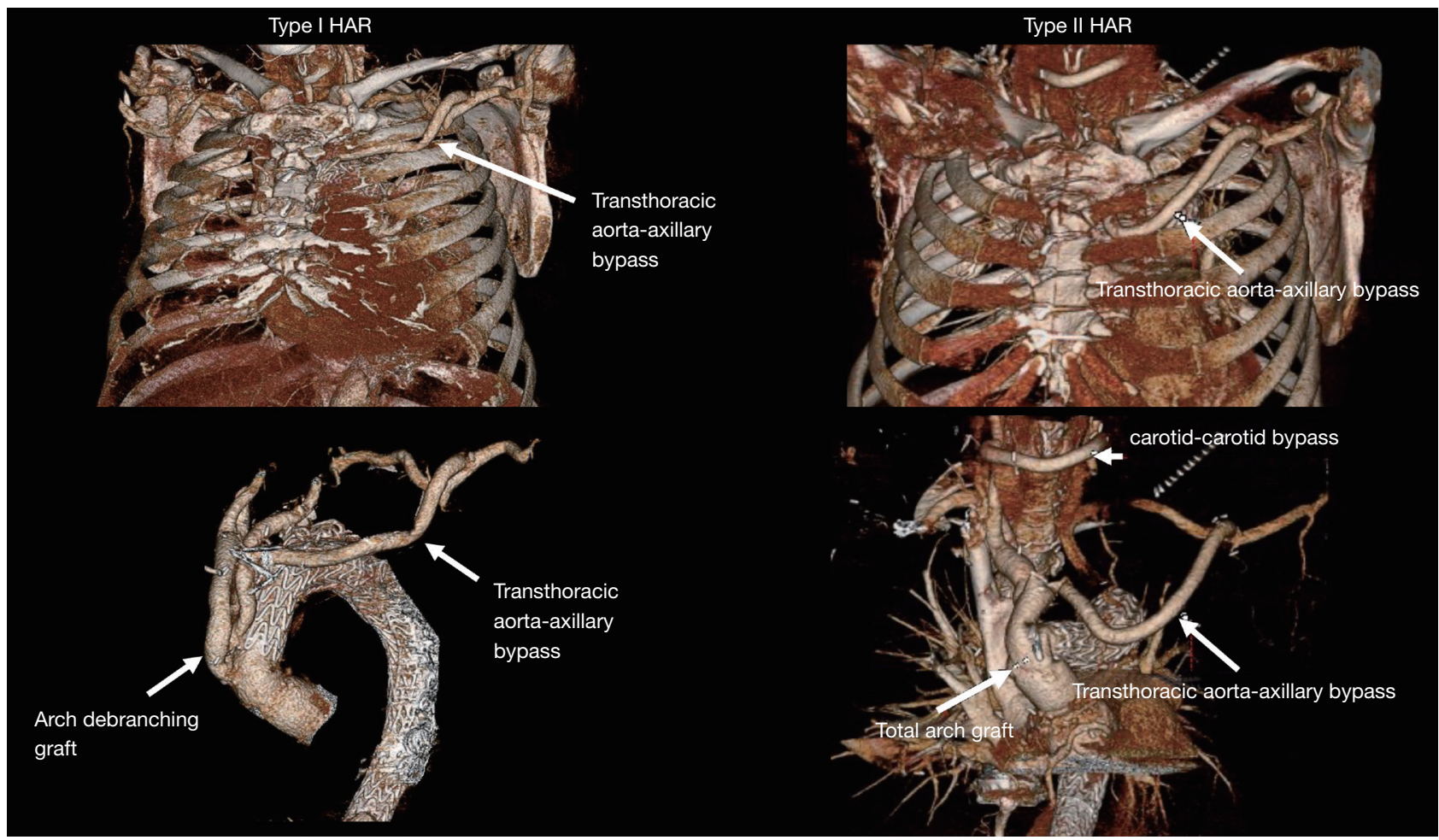

Figure 3 Postoperative CTA 3D-reconstruction images demonstrating examples of type I (left) and type II (right) HAR with transthoracic aorta-axillary bypass, which is performed at the time of the first stage debranching procedure. The type II HAR patient in the right panel also had a right to left carotid-carotid bypass (performed at time of $2^{\text {nd }}$ stage TEVAR), as only the innominate artery was accessible via sternotomy for debranching at the time of the first stage procedure. HAR, hybrid arch repair; TEVAR, thoracic endovascular aortic repair.
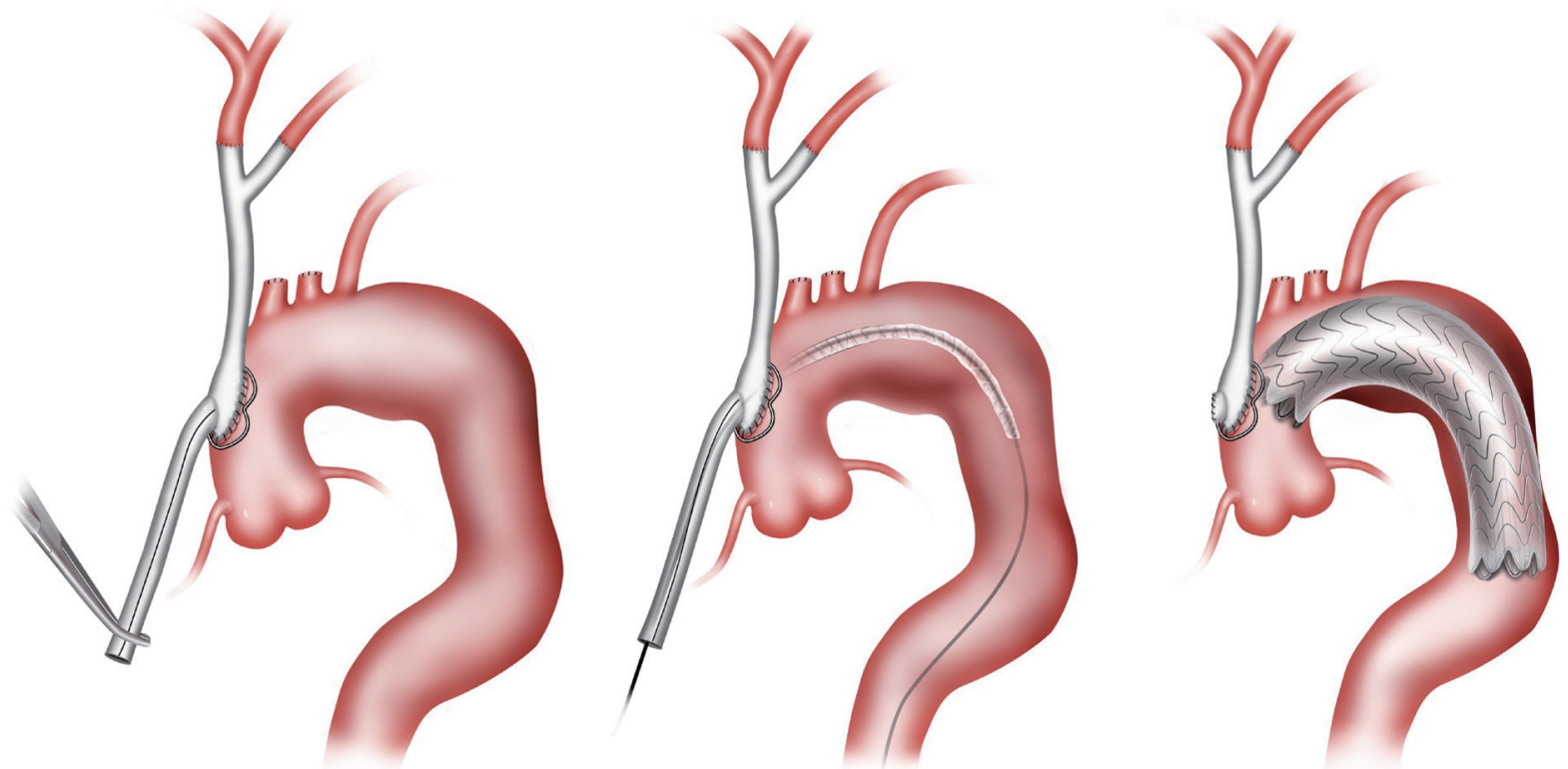

Figure 4 Type I HAR with antegrade stent graft deployment. We reserve the use of this approach to those situations in which the patient has inadequate iliofemoral access for retrograde delivery. HAR, hybrid arch repair. 


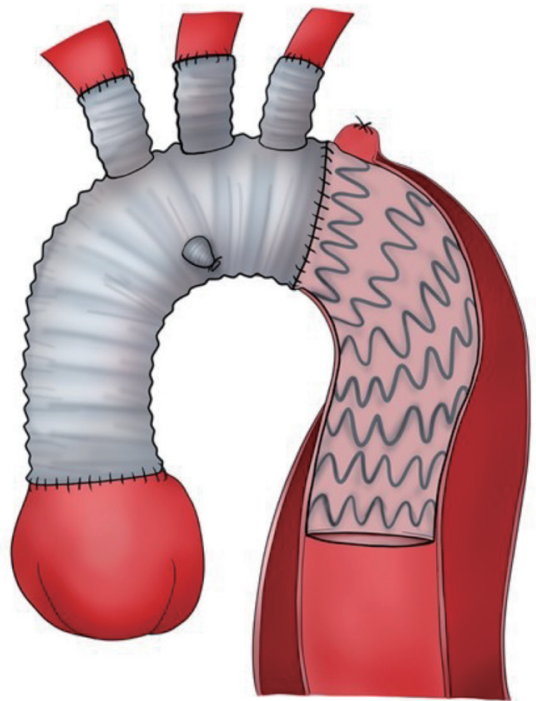

Figure 5 Type III HAR utilizing a frozen elephant trunk. Reproduced with permission from reference (14). HAR, hybrid arch repair.

panel). Perfusion of the innominate artery and cervical debranching bypass graft is maintained via either an offlabel "snorkel" endograft (15) or an investigational branched endograft. This procedure is currently utilized for patients with suitable zone 0 PLZ, yet who are not candidates for the more extensive type I HAR.

\section{Proposed new HAR classification system}

The current classification system has become outdated with the advent of newer technologies, and we propose a new, updated classification system (Figure 7) that is more encompassing with regards to the broad array of options available to practitioners involved in the treatment of aortic arch disease. The new classification system denotes HARs that do not involve opening of the chest cavity as "zone $\mathrm{x}$ " HAR, whereby the $\mathrm{x}$ denotes the PLZ of the most proximal endograft, and HARs which involve opening the chest cavity as "type $\mathrm{x}$ ", similar to the original classification system.

In addition, subscripts are utilized to further differentiate between similar subtypes of HAR. Zone 0 HAR includes zone $0_{\mathrm{s}}$, whereby the arch vessels are debranched using a cervical debranching graft via neck incisions, and flow to the innominate artery and cervical debranching graft maintained via an innominate "Snorkel" endograft. Zone $0_{\mathrm{B}} \mathrm{HAR}$ is similar except that flow to the innominate artery is maintained via a thoracic endograft with a single side Branch for the innominate artery. Zone $1 \mathrm{HAR}$, as described above, is now included in the expanded classification system. Type I HAR includes type $\mathrm{I}_{\mathrm{N}} \mathrm{HAR}$, in which the PLZ is in Native zone 0 , and type $I_{D}$ HAR where the PLZ is previously Dacron-replaced zone 0 . Type II HAR remains unchanged from the current system, whereas type III HAR includes type $\mathrm{III}_{\mathrm{C}}$, denoting Conventional elephant trunk PLZ, and type $\mathrm{III}_{\mathrm{F}}$ to denote arch replacement with FET. The new system does not include total endovascular repair utilizing either two- or three-branch (17) endografts, as these procedures do not involve an open component, save for carotid-subclavian bypass/transposition with dual branch repairs, the latter of which has been considered outside the realm of HAR (7).

\section{Surgical algorithm}

In 2013, our group published an institutional algorithm to guide patient and operative selection for HAR (7). Patients were considered for HAR if they had either highrisk comorbidities or high-risk anatomy, with an important feature of the algorithm being that any decisions about repair strategy should be made by a surgical team with expertise in both open and endovascular techniques. Since the time of that original publication, our institutional algorithm has evolved with greater experience, as well as the availability of new technologies, and an updated algorithm is presented in Figure 8. Of note, for patients with connective tissue disorders or those requiring two-stage open repair, HAR is generally avoided, although HAR may still be considered for connective tissue patients with proximal and distal landing zones in Dacron replaced aorta (18).

\section{Operative considerations}

\section{Preoperative considerations}

For patients undergoing non-sternotomy approaches (zone 0 and $1 \mathrm{HAR}$ ) to HAR, preoperative cardiac evaluation consists of our previously published (19) limited preoperative TEVAR cardiovascular workup including clinical symptoms, resting electrocardiogram and transthoracic echocardiography with further evaluation (stress testing, coronary angiography) undertaken only in the setting of abnormalities on these initial evaluations. For patients undergoing types II or III HAR involving CPB and aortic cross-clamp, coronary angiography is routinely 

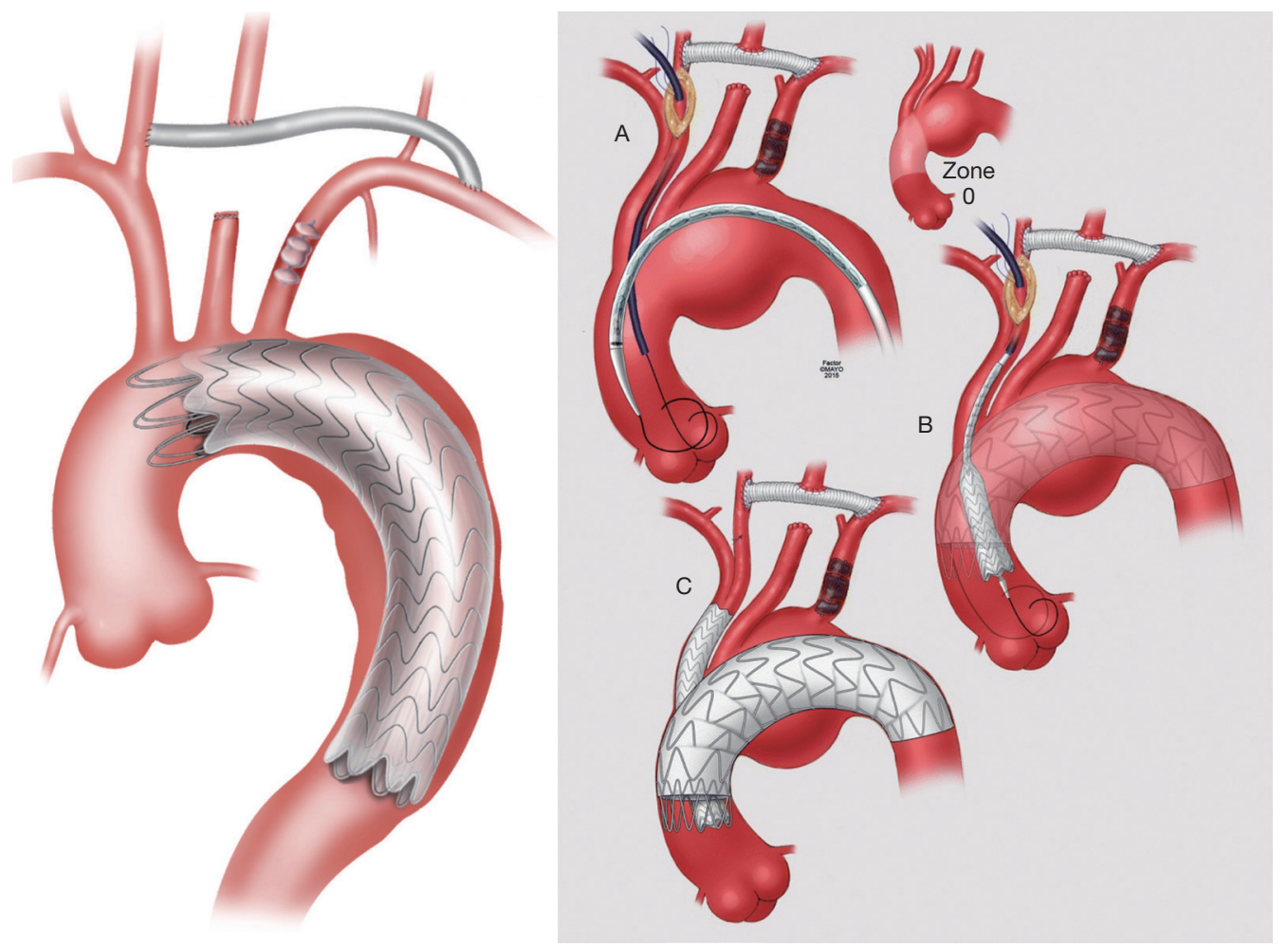

Figure 6 Zone 1 HAR (left panel) with cervical arch debranching (right common carotid-left common carotid-left subclavian artery bypass via neck incisions) with PLZ in zone 1 just distal to the innominate artery. Zone 0 HAR (right panel) involves cervical arch debranching similar to the zone 1 HAR procedure, but with PLZ in zone 0. Perfusion of the innominate artery and cervical debranching bypass graft is maintained via either an off-label "snorkel" endograft, as shown in the figure, or an investigational branched endograft. Right image reproduced with permission from reference (15). HAR, hybrid arch repair; PLZ, proximal landing zone.

performed preoperatively to rule out concomitant CAD similar to other cardiac surgical operations (20). For type I HAR, which does not typically involve aortic crossclamp, coronary angiography is performed on a more select basis similar to zone $0-1$ repairs. Other preoperative testing such as pulmonary function evaluation is carried out when clinically indicated based upon patient history or symptomatology.

\section{Intraoperative considerations}

For all of the various sub-types of HAR, the patient is positioned supine with the arms tucked at the sides and a small bump placed beneath the shoulder blades to assist with neck extension and facilitate exposure of the supraaortic arch vessels. Bilateral radial arterial lines are placed in all cases, and a central venous line is placed for the first stage open operations performed via sternotomy for types
I-III HAR. Electroencephalography neurophysiologic intraoperative monitoring is used to monitor for cerebral ischemia during zone $0-1$ HAR and during the first open stages involving arch debranching for types I-III HAR. Somatosensory and motor evoked potential monitoring is used to monitor for spinal cord ischemia during zone $0-1$ HAR and the second endovascular stages of types I-III HAR (21). Lumbar cerebrospinal fluid drainage is generally avoided for HAR in accordance with our previously published algorithm (22).

\section{Zone 0 and 1 HAR}

Both of these procedures involve cervical arch debranching with right common carotid to left common carotid to left subclavian artery bypass. Two separate cervical incisions, one along the anterior border of the right sternocleidomastoid muscle and the other 1 fingerbreadth above the left clavicle, 


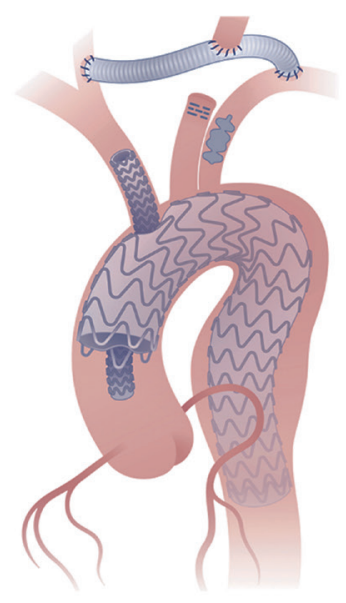

Zone 0 with innominate snorkel $\left(0_{s}\right)$

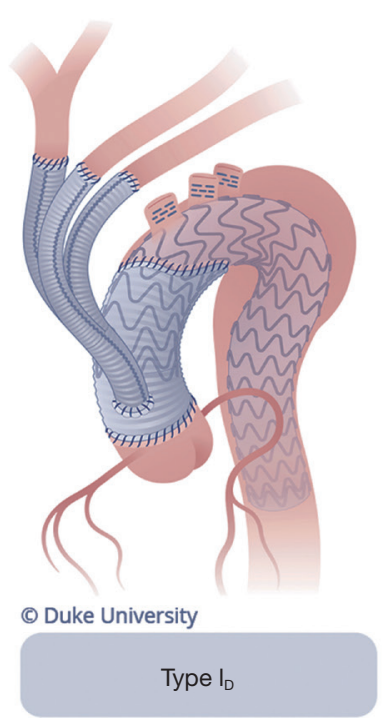

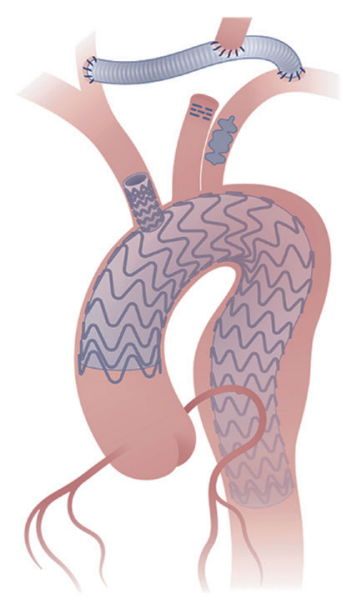

Zone 0 with branched endograft $\left(0_{B}\right)$

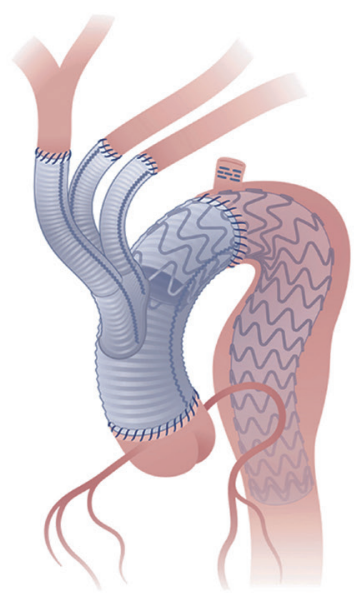

Type II

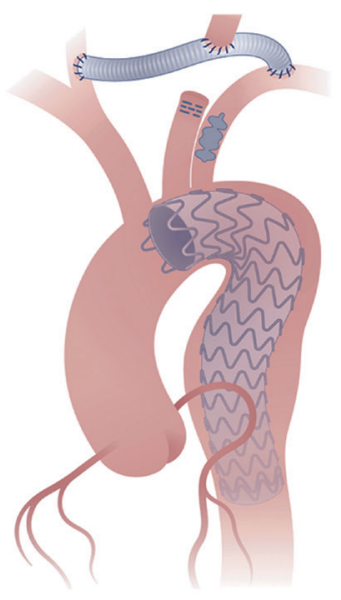

Zone 1
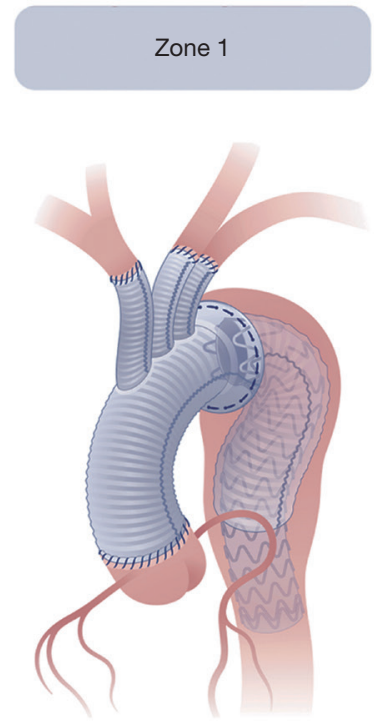

Type III with conventional elephant trunk (IIII)
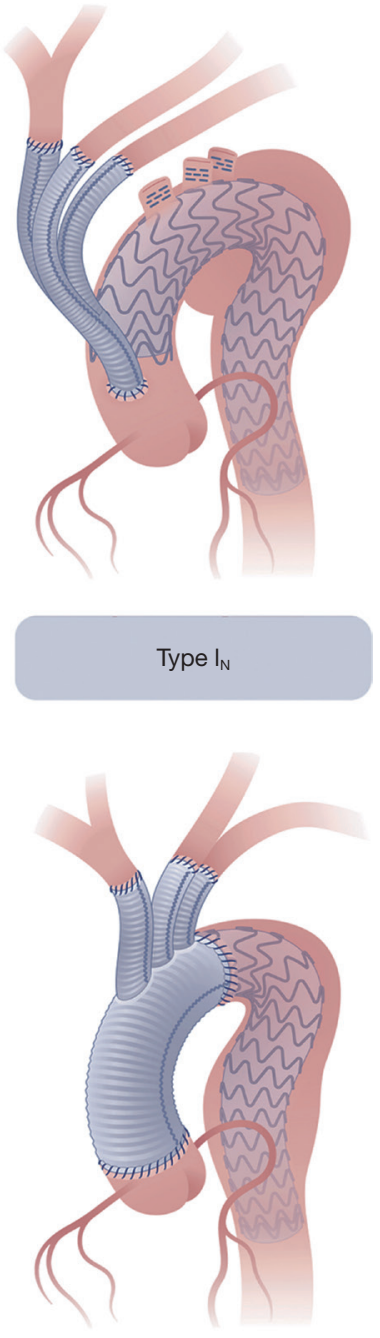

Type III with frozen elephant trunk (III $)$

Figure 7 Proposed new and expanded classification system of hybrid arch repair.

are made to expose the right common carotid, left common carotid, and left subclavian arteries. An $8 \mathrm{~mm}$ non-ringed polytetrafluoroethylene (PTFE) graft is utilized for the carotid-carotid-subclavian bypass. The graft is tunneled anteriorly under the platysma; a retropharyngeal approach, as proposed by others, is avoided due to concerns over dysphagia from posterior esophageal compression (23). The graft is tunneled anteriorly in such a manner that tracheostomy, if needed, is not problematic.

For patients undergoing zone 1 repair, TEVAR is performed during the same operation as the cervical debranching. Similarly, for zone $0_{\mathrm{S}}$ patients utilizing a "snorkel" endograft to perfuse the innominate artery and cervical debranching bypass graft, the TEVAR is performed in a single stage in conjunction with the carotid-carotidsubclavian bypass, thereby allowing the right common carotid artery to be accessed for delivery of the innominate device through the existing right cervical incision, just caudal to the proximal carotid-carotid bypass anastomosis (Figure 6, right panel). The "snorkel" device utilized depends on patient anatomy, but balloon-expandable covered stent grafts oversized by $10 \%$ to the innominate artery landing zone are now most frequently used. The device is advanced retrograde via the carotid sheath into the zone 0 PLZ 


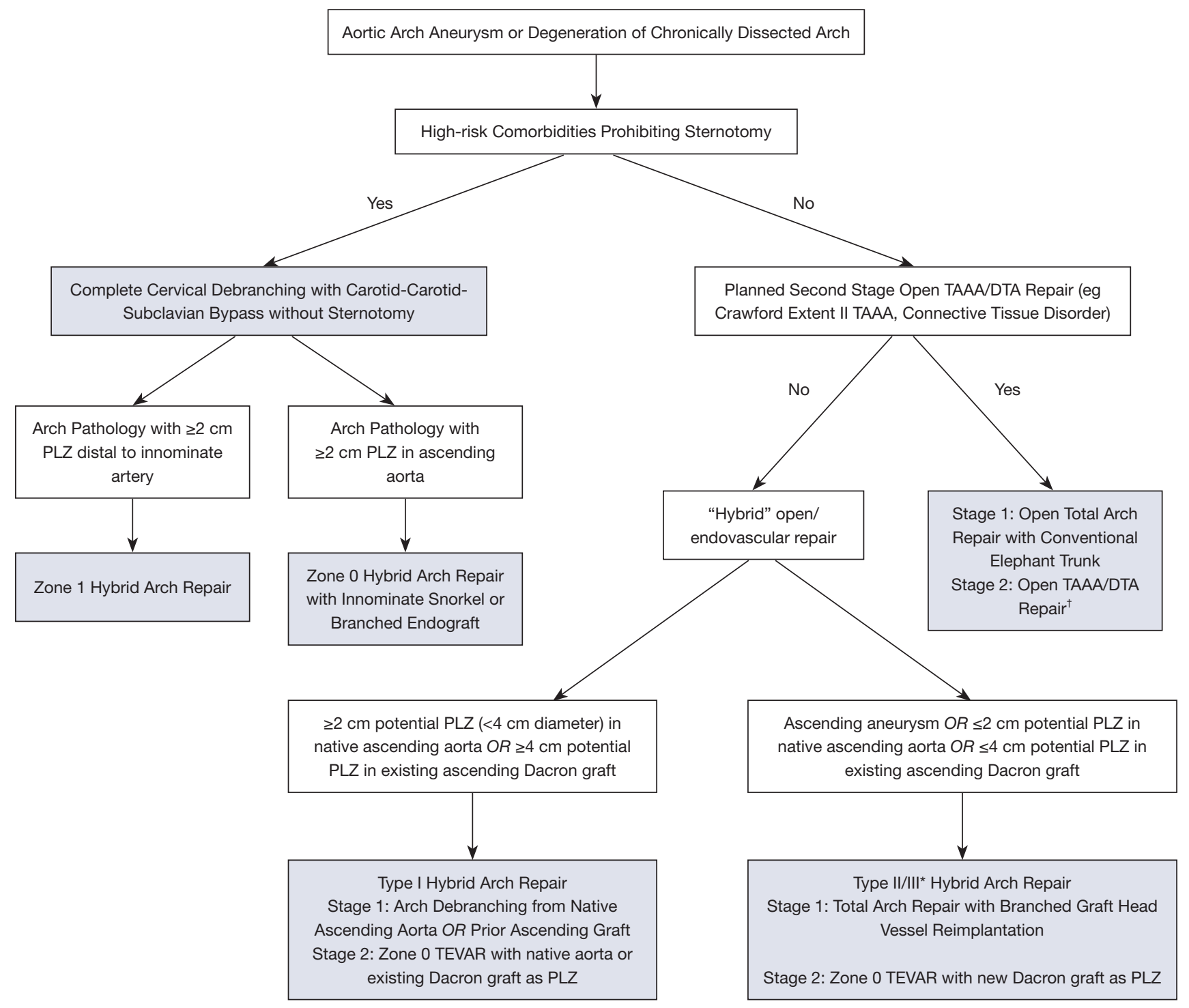

*Institutional preference shifted to Type II HAR in 2016 with commercial availability of grafts designed for this approach
'Single stage arch and descending repair may also be performed through thoracosternotomy (clamshell incision) in select cases
TAAA = Thoracoabdominal Aortic Aneurysm, DTA = Descending Thoracic Aortic Aneurysm, PLZ = Proximal Landing Zone

Figure 8 Algorithm for selecting surgical approach to the patient with an aortic arch aneurysm.

while the thoracic endograft is advanced from the femoral artery through the arch into the ascending aorta. After the "snorkel" and thoracic endografts are positioned within the ascending aorta in parallel positions, with the snorkel device extending proximally just beyond the leading edge of the thoracic device, the devices are simultaneously deployed. For the zone $0_{\mathrm{B}}$ investigational branched endograft cases, the TEVAR is generally staged and performed two-three days following cervical debranching during a single hospital stay due to requirements of the current clinical trials; we would anticipate these cases to be done in a single stage at our institution, similar to the other zone $0-1$ cases, once the devices are fully approved.

\section{Type I HAR}

Also known as the "classic" hybrid arch debranching procedure (24), type I HAR involves head vessel re- 

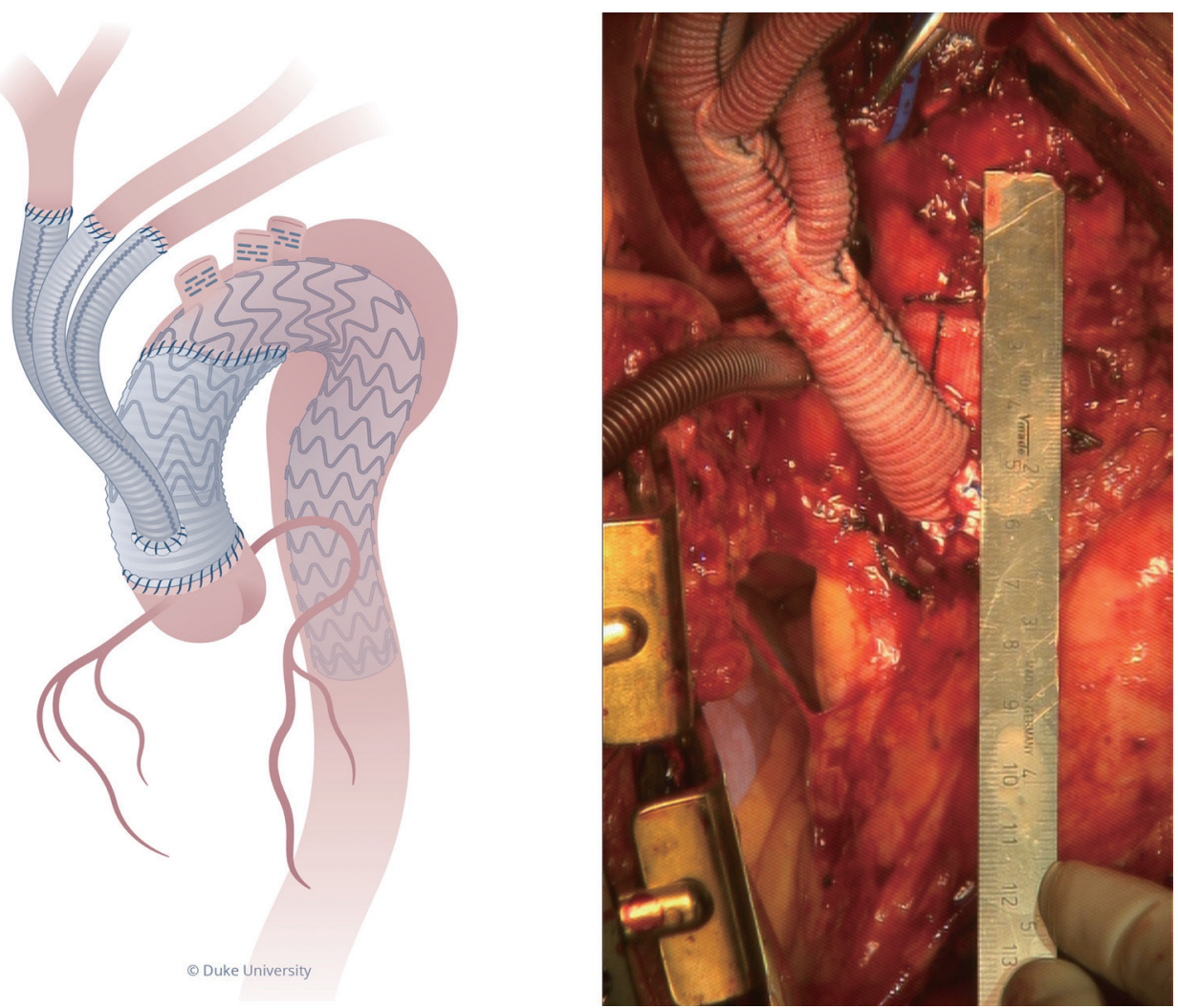

Figure 9 Type $\mathrm{I}_{\mathrm{D}}$ HAR drawing (left) and intra-op photograph (right) demonstrating the 4+ cm PLZ in the existing ascending Dacron graft distal to the arch debranching graft. Illustrated by Megan Llewellyn, MSMI, CMI; copyright Duke University; with permission under a CC BY-ND 4.0 license. HAR, hybrid arch repair; PLZ, proximal landing zone.

implantation (“debranching") via a multi-branched graft from either the native ascending aorta (type $I_{N}$ HAR) or an existing ascending aortic Dacron graft (type $I_{D} H A R$ ), most commonly one which has been placed at the time of prior acute type A dissection repair. The aneurysmal arch pathology is then excluded via endograft(s) with PLZ in the ascending aorta (zone 0 ). This procedure is most commonly utilized at our institution for patients with arch pathology status post prior type A dissection repair (type $\mathrm{I}_{\mathrm{D}} \mathrm{HAR}$ ).

Patient selection for type I HAR is critical if one is to obtain good results. Specifically, for type $I_{N}$ HAR, the procedure is only utilized for patients with a native ascending aorta $<4 \mathrm{~cm}$ in diameter so as to minimize the risk of retrograde type A dissection $(24,25)$. Patients are suitable for type $I_{D}$ HAR only if their existing ascending \pm hemiarch Dacron graft is long enough to accommodate the arch debranching graft proximal anastomosis plus an additional $4 \mathrm{~cm}$ of Dacron graft distal to the debranching graft to serve as PLZ (Figure 9), as prior data has suggested that at least
$4 \mathrm{~cm}$ (twice that of native aorta) of landing zone is required for reliable proximal seal of an endograft within a Dacron graft (26).

A detailed description of our operative technique for type I HAR has been previously published (24), and the reader is referred to that document for additional technical details of the procedure which are beyond the scope of the current review. Briefly, the first stage debranching procedure begins with right axillary cannulation using an $8 \mathrm{~mm}$ side graft technique. Median sternotomy is then performed with the incision extended cephalad along either the right or left sternocleidomastoid muscle to allow dissection and mobilization of the arch vessels, depending on patient anatomy and whether or not all three arch vessels are to be debranched from the front (24). We prefer to mobilize the arch vessels as much as possible prior to full heparinization and institution of $\mathrm{CPB}$. After institution of $\mathrm{CPB}$ and cooling to $34^{\circ} \mathrm{C}$, the native ascending aorta (type $\mathrm{I}_{\mathrm{N}} \mathrm{HAR}$ ) or existing ascending \pm hemi-arch Dacron graft 
(type $I_{D} H A R$ ) is mobilized. In the case of type $I_{D}$ HAR, the ascending graft is mobilized down to as close to the proximal anastomosis at the sinotubular junction as possible, so as to create maximal PLZ length (24). In the native zone 0 setting, a site is chosen on the proximal ascending aorta for the inflow anastomosis of the arch debranching graft that is distant enough from the innominate artery so as to create long segment PLZ. The proximal anastomosis of the arch debranching graft is performed on CPB with the heart empty and beating. Care should be taken to turn the CPB flow down during the application of the partial clamp, especially when clamping a native ascending aorta, so as to minimize the risk of iatrogenic aortic dissection (27). Although the arch debranching and TEVAR portions of the procedure can be performed concurrently using either antegrade or retrograde endograft delivery, performing these cases in two stages during a single hospital stay has evolved as the preferred technique at our institution $(24,28)$.

Early reports with type I HAR, including data from our institution (28), appeared promising, although with increasing experience the limitations of the procedure, especially in the setting of native zone 0 PLZ (type $I_{N}$ HAR), became apparent (7). As a result of these limitations, over the past decade, our group has shifted away from the type $I_{N}$ HAR procedure (29) towards increasing use of type II HAR, and our institutional practice is that even mildly dilated ascending aortas $(>4.0 \mathrm{~cm})$ warrant proximal reconstruction before zone 0 TEVAR with planned arch debranching $(7,11,24)$. However, for patients unsuitable for the more extensive type II/III HAR, type I HAR remains a good option provided the existence of suitable PLZ anatomy (24).

\section{Type II/III HAR}

Type II HAR, including total arch replacement without the creation of an elephant trunk, has become preferred over type III HAR at our institution due to the simpler distal arch anastomosis and shorter period of HCA (11). Details of the operative approach have been previously described (11) and important details are highlighted. Right axillary cannulation and unilateral antegrade cerebral perfusion (ACP) at $12{ }^{\circ} \mathrm{C}$ are utilized along with moderate systemic hypothermia. The total arch replacement portion of the procedure is performed using the "distal first" modification of the Spielvogel technique, whereby the distal arch anastomosis is performed first during the period of unilateral ACP. Lower body perfusion is then reinstituted through a perfusion side branch of the Dacron arch graft, which results in a shorter period of lower body HCA than if the head vessels were reimplanted first. We typically perform the distal arch anastomosis in zone 2, although occasionally in some patients a zone 1 distal anastomosis is utilized if technically easier due to patient anatomy, as in some redo cases after prior type A dissection repair. The LSA and left common carotid arteries are next reimplanted into the corresponding branches of the Dacron arch graft. The innominate artery is reimplanted last, after completion of the proximal aortic work and release of the cross-clamp, as this sequence shortens the cross-clamp time. In nearly half of cases, the LSA arises too far distally on the arch to be reimplanted via sternotomy at the time of the first-stage total arch portion of the procedure, and in this situation the LSA is revascularized, as described above, via left carotidsubclavian bypass at the time of the second stage TEVAR procedure. Alternatively, in select patients in whom carotidsubclavian bypass is anticipated to be technically challenging due to patient anatomy such as obesity, a transthoracic aorta to left axillary bypass can be performed during the first stage repair (Figure 3, right panel).

Similar to type I HAR, the second-stage TEVAR procedure is performed during the same hospital stay. The staged approach during a single hospital stay is preferred given the competing postoperative management strategies with regard to blood pressure after open proximal versus endovascular distal aortic repair (7). Specifically, lower mean arterial pressures are preferred in the early postoperative period after open proximal aortic repair to mitigate bleeding risk, whereas bleeding is generally not an issue after TEVAR, and higher blood pressures are preferred for spinal cord protection (22). Staged repair also reduces the nephrotoxic insult of a prolonged operation involving $\mathrm{CPB}$ and HCA followed by contrast administration, reduces blood loss by limiting the period of heparinization, and allows for patient recovery and medical optimization before the second-stage procedure. Finally, the short (typically three-five days) period between stages during the same hospitalization minimizes concerns about inter-stage aortic events.

\section{Results}

Despite being performed since the early 2000's, the evidence around HAR consists mainly of single center series (level B-C evidence) with no randomized controlled trials. In 2013, Moulakakis and colleagues published a meta-analysis 

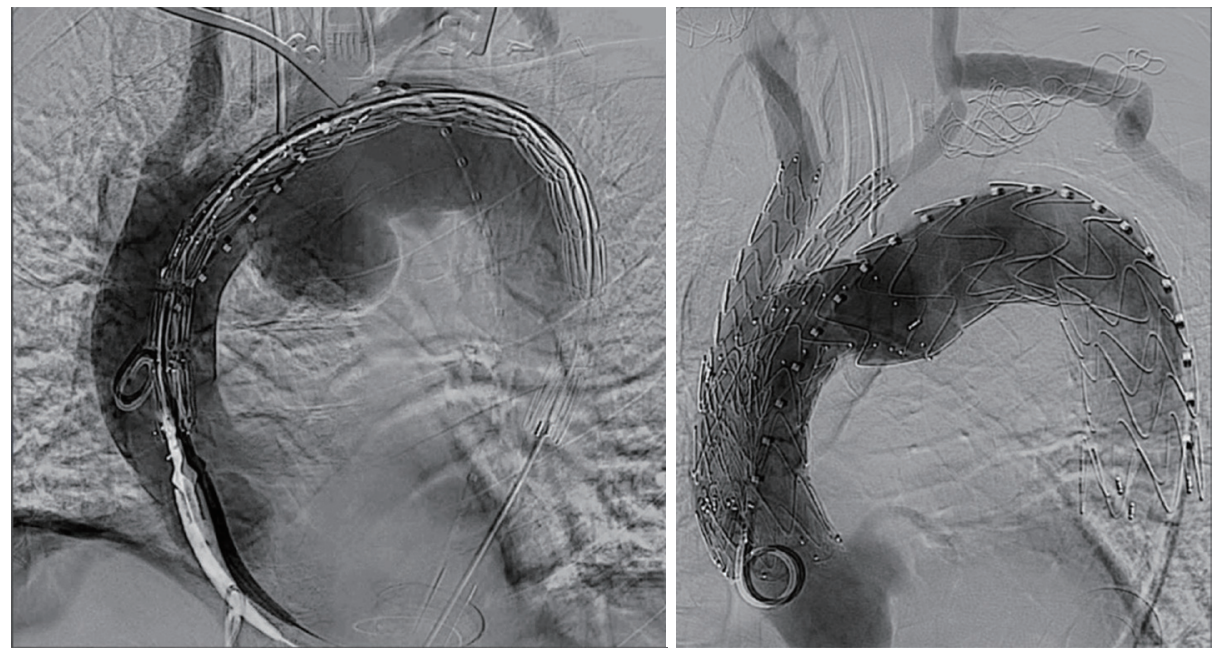

Figure 10 Intraoperative pre- (left) and post- (right) angiograms demonstrating endovascular exclusion of a large saccular mid arch aneurysm in a high-risk patient using an investigational dual branch device. The left subclavian artery has been covered and revascularized via carotid-subclavian bypass.

of HAR, which remains the largest study to date on the topic (30). They analyzed 26 studies including 956 patients undergoing types I-III HAR with supra-aortic debranching. They found a pooled mortality of $11.9 \%$, stroke rate of $7.6 \%$ and permanent paraplegia of $3.6 \%$ with significant heterogeneity of results between studies. They concluded HAR provides a safe alternative to open repair with acceptable short and mid-term results, but that future prospective trials comparing conventional open and hybrid methods are needed.

With regards to the less invasive zone 0 and $1 \mathrm{HAR}$ procedures, Bayfield and colleagues have published a metaanalysis on zone 1 HAR (16), which included twenty studies incorporating 348 patients. In-hospital/thirty-day mortality was $10.1 \%$, stroke $9.5 \%$ and paraplegia $3.8 \%$. Late survival beyond four years postoperatively was $65.9 \%$. Similar to the meta-analysis on types I-III HAR, the authors concluded that zone 1 HAR has evidence for satisfactory short- and long-term morbidity and mortality outcomes and may be considered as an alternative approach in aortic arch disease.

No large data sets exist examining zone $0_{\mathrm{S}}$ HARs, with all studies including a mix of chimney grafts in the various supra-aortic vessels (31-34). Not surprisingly, these procedures have a higher incidence of type 1a gutter endoleak between the innominate snorkel and aortic endograft, and therefore authors have recommended the use of this procedure be strictly limited to high-risk patients without other surgical options (35), which has been our practice as outlined in the institutional algorithm presented in Figure 8.

\section{Future directions}

Since its inception in the early 2000s, HAR has evolved from novel approach to well-established treatment modality for aortic arch pathology in appropriately selected patients. Now some twenty years later, branched endografting has replaced HAR as the "latest and greatest" in the treatment of arch disease, with multiple devices currently in clinical trials (Figure 10) and promising early results. As such, branched endografting stands poised to become an increasingly utilized part of the treatment armamentarium for this population in the future, likely even in many patients who could undergo conventional hybrid or open repair.

\section{Conclusions}

Over the past twenty years, HAR has evolved from an exciting, novel approach to the treatment of arch pathology to something which is done routinely in many aortic centers around the world. As we as aortic surgeons continue to move towards less invasive approaches, both conventional open and hybrid approaches will remain important tools in 
the toolbox for arch repair, although the advent of multibranched arch endografts will almost certainly reduce the extent of open or hybrid repair in many patients and eliminate it altogether in others.

\section{Acknowledgments}

Funding: This work was supported by the National Institutes of Health (5T32HL069749-17 to Dr. A Vekstein).

\section{Footnote}

Conflicts of Interest: GCH: Terumo Aortic: speaker, consultant, clinical trial principal investigator; W. L. Gore and Associates: speaker, consultant, clinical trial principal investigator. The other author has no conflicts of interest to declare.

Open Access Statement: This is an Open Access article distributed in accordance with the Creative Commons Attribution-NonCommercial-NoDerivs 4.0 International License (CC BY-NC-ND 4.0), which permits the noncommercial replication and distribution of the article with the strict proviso that no changes or edits are made and the original work is properly cited (including links to both the formal publication through the relevant DOI and the license). See: https://creativecommons.org/licenses/by-nc-nd/4.0/.

\section{References}

1. Englum BR, He X, Gulack BC, et al. Hypothermia and cerebral protection strategies in aortic arch surgery: a comparative effectiveness analysis from the STS Adult Cardiac Surgery Database. Eur J Cardiothorac Surg 2017;52:492-8.

2. Williams JB, Peterson ED, Zhao Y, et al. Contemporary results for proximal aortic replacement in North America. J Am Coll Cardiol 2012;60:1156-62.

3. Ganapathi AM, Englum BR, Hanna JM, et al. Frailty and risk in proximal aortic surgery. J Thorac Cardiovasc Surg 2014;147:186-191.e1.

4. Czerny M, Fleck T, Zimpfer D, et al. Combined repair of an aortic arch aneurysm by sequential transposition of the supra-aortic branches and endovascular stent-graft placement. J Thorac Cardiovasc Surg 2003;126:916-8.

5. Hughes GC, Sulzer CF, McCann RL, et al. Endovascular approaches to complex thoracic aortic disease. Semin Cardiothorac Vasc Anesth 2008;12:298-319.
6. Bavaria J, Vallabhajosyula P, Moeller P, et al. Hybrid approaches in the treatment of aortic arch aneurysms: postoperative and midterm outcomes. J Thorac Cardiovasc Surg 2013;145:S85-90.

7. Andersen ND, Williams JB, Hanna JM, et al. Results with an algorithmic approach to hybrid repair of the aortic arch. J Vasc Surg 2013;57:655-67; discussion 666-7.

8. Voigt SL, Bishawi M, Ranney D, et al. Outcomes of carotid-subclavian bypass performed in the setting of thoracic endovascular aortic repair. J Vasc Surg 2019;69:701-9.

9. Hughes GC. Commentary: Left subclavian artery revascularization during zone 2 thoracic endovascular aortic repair: Bypass versus transposition? Just do it! J Thorac Cardiovasc Surg 2020;159:1228-30.

10. Bartos O, Mustafi M, Andic M, et al. Carotid-axillary bypass as an alternative revascularization method for zone II thoracic endovascular aortic repair. J Vasc Surg 2020;72:1229-36.

11. Ranney DN, Yerokun BA, Benrashid E, et al. Outcomes of Planned Two-Stage Hybrid Aortic Repair With DacronReplaced Proximal Landing Zone. Ann Thorac Surg 2018;106:1136-42.

12. Dhanekula AS, Sweet MP, Desai N, et al. Aortic arch stenting: current strategies, new technologies and future directions. Heart 2021. (Epub ahead of print).

13. Chung JC, Ouzounian M, Chu MWA, et al. The Evolving Role of Hybrid Arch Repair. Innovations (Phila) 2020;15:506-12.

14. Liu Y, Liang S, Zhang B, et al. Type II hybrid arch repair versus total arch replacement with frozen elephant trunk: a propensity score-matched analysis. Eur J Cardiothorac Surg 2021;60:297-304.

15. Criado FJ. Parallel stent graft techniques to facilitate endovascular repair in the aortic arch. In: Oderich GS. editor. Endovascular Aortic Repair. Switzerland: Springer, Cham; 2017.

16. Bayfield NG, Samuel M, Bayfield AE, et al. Zone 1 Aortic Arch Hybrid Endovascular Repair with Extra-anatomical Bypass: A Meta-analysis. Ann Vasc Surg 2021;72:601-9.

17. Verscheure D, Haulon S, Tsilimparis N, et al. Endovascular Treatment of Post Type A Chronic Aortic Arch Dissection With a Branched Endograft: Early Results From a Retrospective International Multicenter Study. Ann Surg 2021;273:997-1003.

18. Hughes GC. Aggressive aortic replacement for LoeysDietz syndrome. Tex Heart Inst J 2011;38:663-6.

19. Ganapathi AM, Englum BR, Schechter MA, et al. Role 
of cardiac evaluation before thoracic endovascular aortic repair. J Vasc Surg 2014;60:1196-203.

20. Hiratzka LF, Bakris GL, Beckman JA, et al. 2010 ACCF/AHA/AATS/ACR/ASA/SCA/SCAI/SIR/STS/ SVM Guidelines for the diagnosis and management of patients with thoracic aortic disease. A Report of the American College of Cardiology Foundation/American Heart Association Task Force on Practice Guidelines, American Association for Thoracic Surgery, American College of Radiology,American Stroke Association, Society of Cardiovascular Anesthesiologists, Society for Cardiovascular Angiography and Interventions, Society of Interventional Radiology, Society of Thoracic Surgeons, and Society for Vascular Medicine. J Am Coll Cardiol 2010;5 5:e27-e129.

21. Husain AM, Swaminathan M, McCann RL, et al. Neurophysiologic intraoperative monitoring during endovascular stent graft repair of the descending thoracic aorta. J Clin Neurophysiol 2007;24:328-35.

22. Weissler EH, Voigt SL, Raman V, et al. Permissive Hypertension and Collateral Revascularization May Allow Avoidance of Cerebrospinal Fluid Drainage in Thoracic Endovascular Aortic Repair. Ann Thorac Surg 2020;110:1469-74.

23. Yoneyama F, Sato F, Tokunaga C, et al. Postoperative Dysphagia in Debranching Thoracic Endovascular Aortic Repair with Retroesophageal Carotid-Carotid Bypass. Ann Vasc Surg 2017;43:315.e1-4.

24. Hughes GC. Classic hybrid arch debranching (type I hybrid arch repair) without circulatory arrest. Ann Cardiothorac Surg 2018;7:443-50.

25. Williams JB, Andersen ND, Bhattacharya SD, et al. Retrograde ascending aortic dissection as an early complication of thoracic endovascular aortic repair. J Vasc Surg 2012;55:1255-62.

26. Ganapathi AM, Andersen ND, Hanna JM, et al. Comparison of attachment site endoleak rates in

Cite this article as: Hughes GC, Vekstein A. Current state of hybrid solutions for aortic arch aneurysms. Ann Cardiothorac Surg 2021;10(6):731-743. doi: 10.21037/acs-2021-taes-168
Dacron versus native aorta landing zones after thoracic endovascular aortic repair. J Vasc Surg 2014;59:921-9.

27. Williams ML, Sheng S, Gammie JS, et al. Richard E. Clark Award. Aortic dissection as a complication of cardiac surgery: report from the Society of Thoracic Surgeons database. Ann Thorac Surg 2010;90:1812-6; discussion 1816-7.

28. Hughes GC, Daneshmand MA, Balsara KR, et al. "Hybrid" repair of aneurysms of the transverse aortic arch: midterm results. Ann Thorac Surg 2009;88:1882-7; discussion 1887-8.

29. Benrashid E, Wang H, Keenan JE, et al. Evolving practice pattern changes and outcomes in the era of hybrid aortic arch repair. J Vasc Surg 2016;63:323-31.

30. Moulakakis KG, Mylonas SN, Markatis F, et al. A systematic review and meta-analysis of hybrid aortic arch replacement. Ann Cardiothorac Surg 2013;2:247-60.

31. Hogendoorn W, Schlösser FJ, Moll FL, et al. Thoracic endovascular aortic repair with the chimney graft technique. J Vasc Surg 2013;58:502-11.

32. Bosiers MJ, Donas KP, Mangialardi N, et al. European Multicenter Registry for the Performance of the Chimney/Snorkel Technique in the Treatment of Aortic Arch Pathologic Conditions. Ann Thorac Surg 2016;101:2224-30.

33. Pecoraro F, Lachat M, Cayne NS, et al. Mid-term Results of Chimney and Periscope Grafts in Supra-aortic Branches in High Risk Patients. Eur J Vasc Endovasc Surg 2017;54:295-302.

34. Voskresensky I, Scali ST, Feezor RJ, et al. Outcomes of thoracic endovascular aortic repair using aortic arch chimney stents in high-risk patients. J Vasc Surg 2017;66:9-20.e3.

35. Banno H, Mutsuga M, Sugimoto M, et al. Midterm Outcomes of Zone 0 Antegrade Endograft Implantation During Type I Hybrid Aortic Arch Repair. Eur J Vasc Endovasc Surg 2021;61:938-44. 